\title{
Los bordes de la ciudad y la simulación del crecimiento urbano con autómatas celulares
}

\author{
M. C. Polidori, M. V. P. Saraiva, \\ O. M. Peres, C. P. Toralles y F. Tomiello
}

\section{Resumen}

Las simulaciones de crecimiento urbano utilizando autómatas celulares han mostrado diferencias en los bordes de expansión en comparación con otras partes de la ciudad, con concentración en las interfaces urbanas y rurales y las zonas más urbanizadas con las menos urbanizados. Esta concentración tiende a delimitar frentes de expansión en forma de línea, con borde de determinación y comportamiento diferente del resto del sistema, donde se puede observar simultáneamente concentración y exclusión, células con alto potencial para la conversión, y baja concentración de factores urbanos positivos. Estos frentes de expansión se están estudiando con simulaciones en las pequeñas ciudades del sur de Brasil, a través del software CityCell, desarrollado por el Laboratorio de Urbanismo, Facultad de Arquitectura y Urbanismo de la Universidad Federal de Pelotas. Por lo tanto, este documento presenta una visión general de las simulaciones de crecimiento y los resultados que se han logrado.

Palabras clave: Bordes de la ciudad. Crecimiento urbano. Concentración. Exclusión.

\section{Abstract}

Simulations of urban growth using cellular automatons have shown differences in the comparison of expansion edges to other parts of the city, concentrated in the interfaces of the urban with the non-urban and the most urbanized with the least urbanized. This concentration tends to demarcate expansion fronts in shaped line, determining a border with different behavior from the rest of the system, in which it is observed simultaneous concentration and exclusion, with cells of high potential for transformation and low concentration of urban facilities. These expansion fronts are being studied with simulations in small towns of southern Brazil, through the City Cell software, developed by the Laboratory for Urbanism in the Faculty of Architecture and Urbanism at the Federal University of Pelotas. Thus, this paper presents an 
overview about the simulations of growth and the results that have been achieved.

Keywords: edge city; urban growth; concentration; exclusion.

M. C. Polidori, (mauricio.polidori@terra.com.br); M. V. P. Saraiva, O. M. Peres, C. P. Toralles e F. Tomiello. Universidade Federal de Pelotas, Brasil. 


\section{INTRODUCCIÓN}

Las simulaciones han sido considerados como importantes herramientas para el trabajo en el campo del urbanismo, especialmente cuando implican la interacción entre lo físico, social y ambiental (Alberti, 1999). Las simulaciones pueden proporcionar a los investigadores escenarios hipotéticos de las ciudades reales, fomentar el descubrimiento y la asistencia en la formulación de teorías (Axelrod, 1997). Técnicas computacionales, las cuales pueden ser agrupadas bajo el concepto de geo computación, están siendo cada vez más utilizadas en los estudios de simulación y modelado urbano (Burrough, 1998; Batty, 1998).

Un aspecto de las ciudades que pueden investigarse con el uso de la simulación es su crecimiento, que incluye la captura dinámica del cambio, teniendo en cuenta el tiempo y modos espaciales de representación. En este trabajo, los cambios se toman como algo que surge de la interacción entre la estructura urbana, la estructura social y el medio ambiente natural, modelados mediante dinámica computacional con autómatas celulares (O'Sullivan y Torrens, 2000). El tiempo será incorporado como una sucesión de estados, donde los mecanismos de retroalimentación positiva y negativa interactúan e influyen en los estados sucesivos. El modo de representación adoptado es la de una cuadrícula en células que representan espacios discretos y contienen atributos físicos, naturales e institucionales (Polidori, 2004).

El crecimiento de la ciudad se compone de dinámicas internas y externas, la primera de las cuales se produce a través de la ocupación de los espacios vacantes o sustituciones de las edificaciones existentes, mientras que la segunda opera mediante la conversión de tierras no ocupadas en los nuevos espacios urbanos, con un proceso complejo, organizado por sí mismo y emergente. La idea de la complejidad se deriva de la comprensión de la ciudad y sus cambios como algo que incluye múltiples interferencias y agentes, con varias relaciones posibles entre ellos, que se producen iterativamente con retroalimentación y compuesto, con el fin de acelerar o frenar cambios. El concepto de auto-organización se asocia con la idea de la ausencia de un elemento de control central de la ciudad (que podría dictar sus transformaciones), siendo el resultado lógico de crecimiento urbano de la ciudad, donde sus atributos, que se pueden encontrar en su morfología y el sustrato natural y la sociedad, se encargan de grabar y guardar los cambios. La idea de emergencia aparece como el entendimiento de que el proceso iterativo, complejo y auto organizado de la ciudad plantea notables patrones de ordenamiento espacial, que se pueden ver en la morfología y en el procesamiento de preexistencias urbanas y naturales (Portugali, 1997; Johnson, 2003; Polidori 2003). 
Una de las emergencias o las pautas de ordenamiento territorial que se pueden estudiar a través de simulaciones de crecimiento urbano es la formación de las periferias urbanas, que pueden estar asociados con el proceso conocido como la segregación socio-espacial. El concepto de las periferias urbanas de este trabajo está vinculado a los estudios de la morfología urbana, en que predomina la idea de una línea fronteriza en la ciudad, con tamaños que van desde manzanas hasta barrios enteros donde se concentra la población de bajos ingresos, así como la urbanización y los edificios precarios (Benévolo, 1975, el Bayón y Gasparini, 1977). La idea de la segregación señala la diferenciación espacial observable entre los barrios de tugurios urbanos y otras áreas, especialmente representados por el centro tradicional y los barrios ocupados por los extractos de altos ingresos (Villaça, 2001).

Por lo tanto, se puede suponer que el proceso de urbanización ha estimulado la segregación socio-espacial, mientras que la sociedad urbana trabaja selectivamente la transformación de los lugares de acuerdo a sus necesidades, las disputas y conflictos. En cuanto al patrón de segregación en la metrópoli brasileña, se ha observado que los ingresos de las capas superiores tienden a estar ubicadas en el centro tradicional y los barrios considerados nobles, dotados con más servicios urbanos, mientras que la periferia está ocupada por los más pobres en la ciudad, que se encuentran en las zonas de menor calidad, entre las que destacan los bordes de la ciudad (desde Villaça, 2001). Del mismo modo, cerca de los límites de la ciudad son peores servicios de transporte público, electricidad, agua potable, servicios sanitarios, de ocio, la seguridad y otros aspectos de la vida urbana.

Sin embargo, parece que es precisamente en el borde de la ciudad que se puede observar una gran concentración de potencial de crecimiento y cambio urbano. El área central de la ciudad, ya construida y apropiada por los grupos sociales de mayores ingresos, parece ofrecer mayor dificultad o lentitud al cambio, que implica la eliminación de preexistencias y sustitución de grandes inversiones en infraestructura. Por otro lado, la periferia urbana es un lugar estratégico para el surgimiento de nuevas políticas urbanas, lo que aumenta en función del encuentro con el espacio urbano y rural, la proximidad de los recursos naturales, el valor más bajo de la tierra y la tasa de crecimiento espacial superior a las demás regiones de la ciudad (Polidori y Bachilli, 2007).

Este trabajo está dedicado al estudio del borde exterior de la ciudad y la posibilidad de las interfaces internas donde se esperan cambios que se producen debido al crecimiento de la ciudad y la presencia de las periferias urbanas, asociadas con el proceso de segregación socio espacial. Como se muestra a continua- 
ción, estos bordes pueden tener características especiales, lo que permite formular la hipótesis de que ocurre simultáneamente en el borde, exclusión y concentración, que se puede describir por rarefacción de los valores de centralidad y el aumento de cambio potencial. Para probar esta hipótesis son realizadas simulaciones de crecimiento urbano utilizando el software CityCell, que opera en ambiente de autómatas celulares, propuesto originalmente por Polidori (2004) con el apodo de $\mathrm{SACl}$ - Simulador del Medio Ambiente de la Ciudad, actualmente desarrollado por el Laboratorio de Urbanismo FAUrb - LabUrb por Saraiva, Polidori, Toralles y Peres (2012).

\section{MODELOS, AUTÓMATAS CELULARES Y SIMULACIÓN DEL CRECIMIENTO URBANO CON EL SOFTWARE CITYCELL}

Los modelos son representaciones simplificadas de la realidad (Almeida, Câmara y Monteiro, 2007), con el objetivo principal de representar cierto sistema para incluir los principales aspectos de las teorías subyacentes, y ayudar en la comprensión de los procesos que rigen su dinámica (Batty, 2009). Los modelos se han utilizado con éxito en la representación y simulación de sistemas complejos y dinámicos, como las ciudades (Batty, 1998), permitiendo que los procesos urbanos se reproducen en el laboratorio, lo que permite realizar experimentos que serían imposibles en la práctica, dada la incapacidad para manipular a las personas 0 estructuras físicas reales (Krafta, 2009). Aunque inicialmente fue considerado como representaciones puramente materiales de la realidad, modelos obtenidos a través de los sistemas informáticos han hecho posible representar a la ciudad de manera simbólica, basado en la traducción de ciertas características en lenguaje matemático. De esta manera, el uso de modelos urbanos se convierte en complementario a la idea de la teoría, donde las computadoras actúan como una especie de laboratorio y el fenómeno urbano, después de ser capturado, se puede reproducir experimentalmente mediante simulaciones (Echenique, 1975, Batty, 2009).

El uso de autómatas celulares - AC está conectado con el desarrollo de la vida informática, la inteligencia artificial y (Torrens, 2000a), que ha sido avistado desde los primeros trabajos de John von Neumann (precursor de la teoría de la computación y juego) y Stanislaw Ulmam (uno de los primeros desarrolladores de técnicas de Monte Carlo), en la década de 1940, a los argumentos de Stephen Wolfram (referencia en informática teórica contemporánea, matemático y erudito en AC, como se sugiere en Greco, 2002). Antes de eso, en los años 30, Alan Turing ya estaba pensando en "Universal Turing Machine", como más tarde se supo, porque es un hipotético autómata, que impulsó a pensar en los años 40, dando lugar a la creación de computadoras que siguió los mismos principios lógicos y conceptua- 
les. La idea era crear un "equipo universal", que no necesita ser reconstruida, sólo reprogramada; una tarea imaginada para esta máquina sería "usted mismo duplicado" (Maddox, 1983), lo que importa tanto al campo de la fenómenos naturales como a la ciencia social y espacial (Axelrod, 1997). Las "máquinas de Turing" muestran una lista de reglas para los AC, aplicables a través de ciertos ciclos en el tiempo, en cuyos intervalos (o estados) y procesos (o dinámica) que el mundo podría ser examinado.

Una definición objetiva de AC ha sido desarrollada por Wolfram Stephem desde los años 80 del siglo XX, que dice que AC son representaciones de sistemas físicos a través de las células, cuyas características son especificadas por los valores de las células en cada estado en el que el espacio y el tiempo son valores discretos. En el AC, los valores que cada variable asume se ven afectados por los valores de su vecindario en el tiempo anterior, y los nuevos valores se actualizan simultáneamente, de acuerdo con normas locales (Wolfram, 1983).

Las posibilidades para representar procesos dinámicos con AC se han utilizado como ayuda en la resolución de muchos problemas urbanos y ambientales, tales como el espacio (Ward, Phinn y Murray, 2000). AC son ventajosas para su uso en simulaciones urbanas, especialmente en comparación con los modelos tradicionales (Xie y Batty, 1994), debido a las posibilidades de integración de los requisitos de eficiencia con equidad, así como para incorporar espacialidad absoluta (o cartesiano) y relativa (o Leibnitziana) y sobre todo representar procesos dinámicos (Torrens 2000b).

Con la función de representar a la ciudad y su proceso de transformación, los AC deberían tener adaptado algunas de sus características, que se llama autómatas celulares ampliado con los siguientes ajustes principales (Batty y Xie, 1994; Wu, 1996, White et al 1999; Engelen y White, 2000): a) la red no se considera continuo porque las células en un lado del borde no se consideran célula vecina desde otro lado, b) las células se han adaptado a la escala de tamaño del espacio que está destinado a representar, así como sus estados representan los atributos espaciales, que pueden ser registradas por los números fraccionarios; c) el barrio no se limita a los tradicionales cuatro $u$ ocho células adyacentes al centro de la célula (el barrio de von Neumann y Moore, respectivamente), y puede ser regulado por diversos radios asociadas y áreas de cobertura de las funciones, d) las normas se dedican a replicar procesos reales que tienen lugar en la ciudad y el paisaje, los conceptos y las teorías sobre las transformaciones urbanas y ambientales e) el tiempo se transcribe en tiempo real, lo que representa la evolución urbana y los horizontes de los escenarios que se pretende simular. 
En este trabajo se utilizó el modelo de simulación del crecimiento urbano originalmente redactado por Polidori en 2004, que se dedica al estudio de los cambios en el área urbanizada de la ciudad, teniendo como entorno una base espacial con células cuadradas, tratadas como una cuadrícula bidimensional de un Sistema Información Geográfica - SIG, con las características de funcionamiento de un autómata celular (Batty, Couclelis y Eichen, 1997). Se supone entonces que cada par de células que tienen una característica urbana desarrolla una tensión, tal como nos modelos de centralidad y performance (Krafta, 1994; Polidori, Granero y Krafta 2001). Este tensión se calcula por el producto de la carga total en cada célula a plena carga de la otra que son accesibles, similar a lo que ocurre en los modelos de interacción espacial (Wilson, 1985; Torrens, 2000b), pero sin limitaciones en cuanto al origen y destino. Estas tensiones se distribuyen de modo axial, polar y difuso (ver Figura 1) y su suma en cada célula es llamada de centralidad celular. Secuencialmente, se supone que el potencial de crecimiento es la diferencia entre la centralidad de cada célula con su entorno, lo que puede resultar en un crecimiento interno o externo a las células urbanas preexistentes. Así, las células que tienen uno mayor potencial de crecimiento son las que tienen menos centralidad interior, combinada con mayor centralidad del entorno (Polidori, 2004).

Este modelo de simulación del crecimiento urbano, originalmente llamado por Polidori de SACl - Ciudad Simulador de Medio Ambiente en 2004, ahora se implementa través del software CityCell, como se ha indicado anteriormente. Una de las habilidades desarrolladas en CityCell es la representación y la extracción de los bordes de las ciudades en las simulaciones de crecimiento en una operación de álgebra de mapas, siendo el uso de estos recursos una de las bases de este trabajo, tal como aparece en el epígrafe siguiente.

Figura 1: Diagrama en forma de AC: a) células incluidas en la distribución del tipo axial; b) células incluidas en la distribución de tipo axial, con buffer igual a un; c) células incluidas en la distribución del tipo polar; d) hipótesis de células incluido en la distribución difusa.
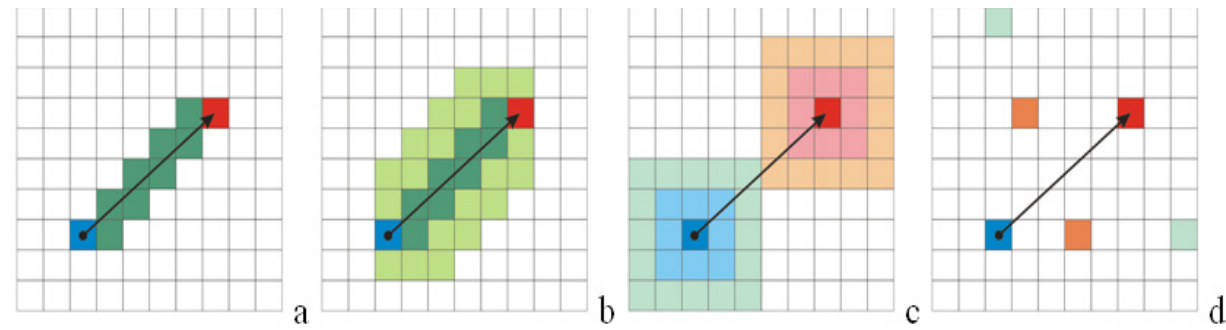

Fuente: Elaboración propia. 


\section{ESTUDIO DE CASO CON LAS CIUDADES DE ARROIO GRANDE Y JA- GUARÃO EN EL SUR DE BRASIL}

Para verificar la ocurrencia de la formación de los bordes de las simulaciones de crecimiento urbano llevó a cabo un experimento en las ciudades de Arroio Grande y Jaguarão, en el sureño estado de Rio Grande do Sul, Brasil, debido a su tamaño ya la disponibilidad de información adecuada, por el SIG realizado por las asociaciones de la Universidad con el Ayuntamiento. Las ciudades son descritas por unas cuadrículas $200 \mathrm{~m}$, que interactúan en el entorno natural y urbano. La primera representada por el área efectivamente urbanizada y el segundo por los recursos de vegetación y agua. Los detalles sobre el funcionamiento del modelo de simulación se pueden conseguir en el trabajo Polidori (2004) ${ }^{1}$.

Las simulaciones se realizaron para un tiempo de cuarenta años, con una iteración para cada año. El modelo fue calibrado para aproximarse cuantitativamente y cualitativamente cómo las ciudad ha crecido en las últimas dos décadas, por la regulación de las tasas de crecimiento y los tipos de ocupación espacial del espacio axial, polar y dispersiva. Los resultados, que ciertamente pueden ser mejor visualizados en animaciones digitales se resumen en tres iteraciones en las figuras 2 y 3 , para el caso de Arroio Grande y las figuras 4 y 5 en el caso Jaguarão. De estas simulaciones se pueden hacer las observaciones principales:

a) la centralidad sigue siendo baja en los bordes de la ciudad a lo largo de la simulación; sin embargo, conforme aumenta el tamaño de la ciudad, las zonas de menor centralidad se desplazan a regiones más alejadas del centro tradicional;

b) el centro tradicional sigue siendo estable a lo largo de la simulación, siendo el portador de privilegios no afectado por los cambios en la periferia; c) el potencial de crecimiento se concentra en los bordes externos de las ciudades, para el caso de Arroio Grande e internos para el caso de Jaguarão desde el comienzo de la simulación, que se vuelve más pronunciado a medida que avanza el tiempo;

d) este potencial se presenta con dos formas predominantes: de forma difusa y fragmentada o como una línea con varios máximos, como la forma de un collar.

\footnotetext{
${ }^{1}$ Consultar: http://www.ufpel.edu.br/faurb/laburb/
} 


\section{CONCLUSIONES: BORDE, EXCLUSIÓN, CONCENTRACIÓN E IRREGULA- RIDADES}

a) Hay un efecto de borde urbano: de hecho las simulaciones demostró la formación de un borde diferente del resto de la ciudad en el proceso de crecimiento; como Polidori había sugerido en 2004 , la dinámica de las transformaciones urbanas indica que el potencial de crecimiento se concentra en las interfaces de las zonas urbanas con las zonas rurales, como se ve en el caso de Arroio Grande y con la más urbanizada y menos urbanizada, como se ve en el caso de Jaguarão; esta concentración tiende a delimitar frentes de expansión en forma de línea, mediante la determinación de borde con comportamiento diferente del resto del sistema; la aparición de efecto de borde en la ciudad trae revitalización del proceso de crecimiento, que se puede utilizar en los planes y proyectos urbanos.

b) Exclusión y concentración son parciales en el borde de la ciudad: la hipótesis planteada en este artículo, que ocurre simultáneamente exclusión y concentración de borde está parcialmente confirmada; si por un lado se confirma la rareza centralidades de esta frontera, por otro lado se puede observar que la concentración de potencial en el borde aparece con valores altos o moderados intercalados; este resultado puede explicarse en parte por el sustrato natural, que opera como un campo irregularidades, como se ha demostrado en otros estudios (Polidori 2004; Polidori y Krafta 2005, Peres y Polidori, 2011).

c) La constitución interna del borde de la ciudad es irregular: los resultados obtenidos en los experimentos de este trabajo, acumulados con los estudios previos realizados por el personal del Laboratorio de Urbanismo FAUrb, usando autómatas celulares (Peres y Polidori, 2009 y 2010; Peres, y Polidori Saraiva, 2010; Saraiva y Polidori, 2011; Peres, Saraiva y Polidori, 2011) sugieren que el borde de la ciudad tiene características de irregularidad, de forma similar a los sistemas fuera del equilibrio, sin embargo, las características más específicas de este comportamiento no se conocen, el que está sujeto a ser investigado en el futuro. 
Figura 2: a, b, c) la evolución de la distribución de la centralidad (gradiente azul) en la ciudad de Arroio Grande, en las iteraciones 1, 20 y 40, respectivamente; d, e, f) la evolución de la distribución de la centralidad en la ciudad de Arroio Grande, con bordes de demarcación (magenta) en la iteración 1,20 y 40, respectivamente.

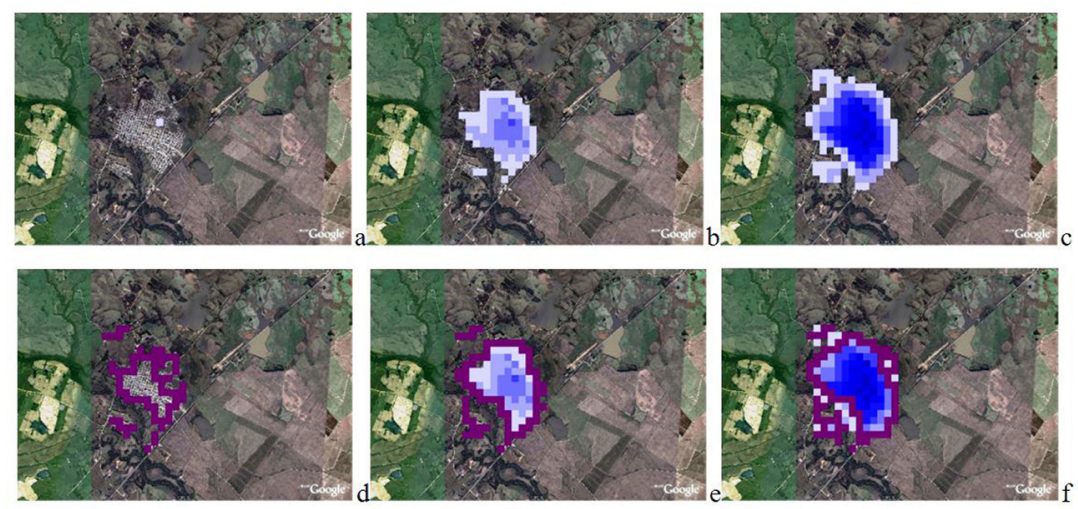

Fuente: Elaboración propia.

Figura 3: a, b, c) los cambios en la distribución potencial (gradiente de color naranja) en la localidad de Arroio Grande, en iteraciones 1, 20 y 40, respectivamente; d, e, f) los cambios en la distribución potencial (gradiente de color naranja) en Ciudad de Arroio Grande, marcando los bordes

(magenta) en iteraciones 1, 20 y 40, respectivamente.
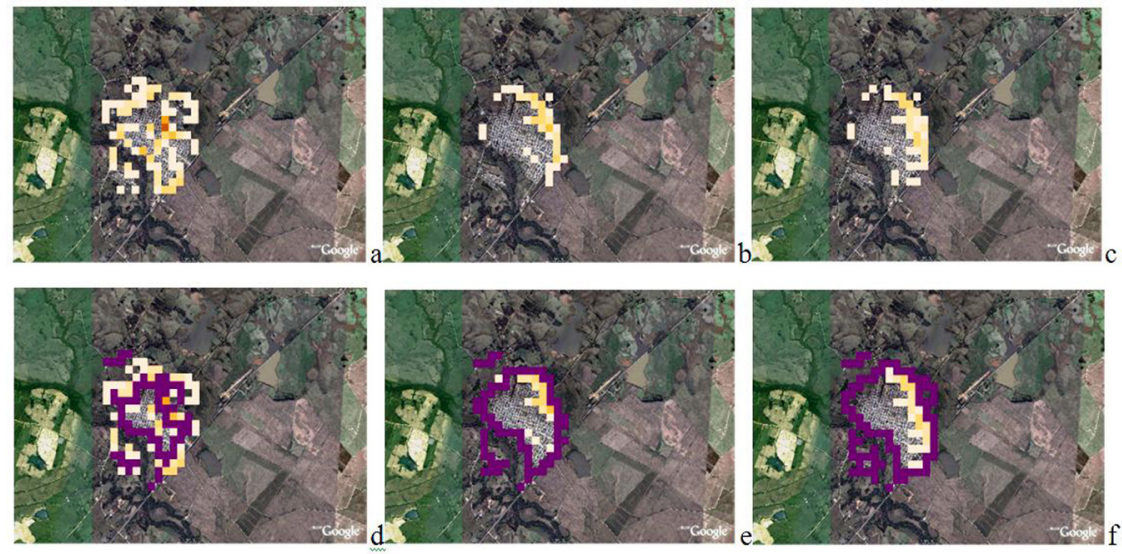

Fuente: Elaboración propia. 
Figura 4: a, b, c) la evolución de la distribución de la centralidad (gradiente azul) en la ciudad de Jaguarão en iteraciones 1, 20 y 40, respectivamente; d, e, f) la evolución de la distribución de la centralidad en la ciudad de Jaguarão con bordes de demarcación (magenta) en iteraciones 1, 20 y 40 , respectivamente.
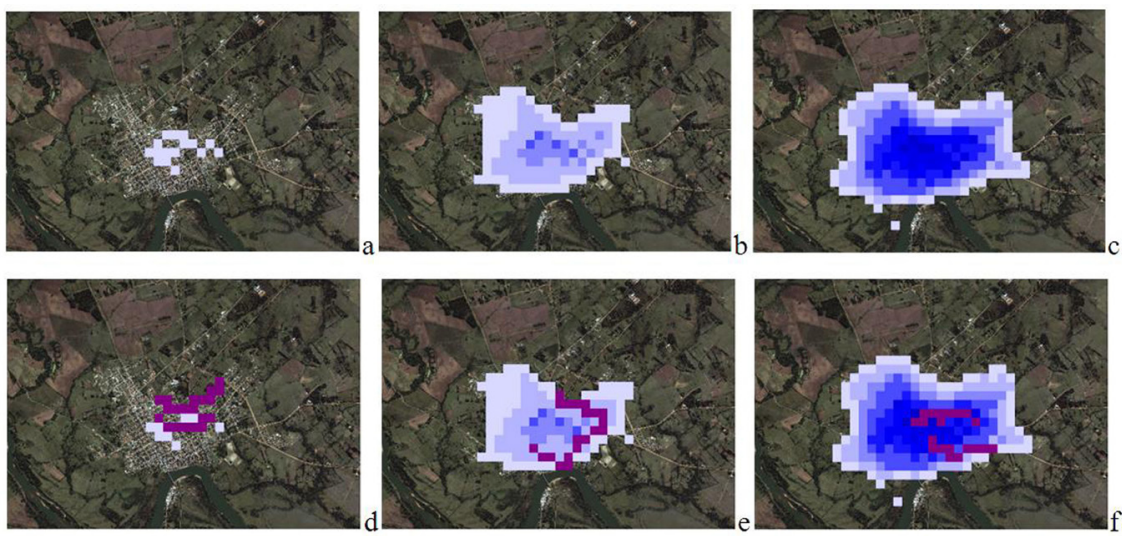

Fuente: Elaboración propia.

Figura 5: a, b, c) los cambios en la distribución potencial (gradiente de color naranja) en la ciudad de Jaguarão, en iteraciones 1, 20 y 40, respectivamente; d. e, f) los cambios en la distribución potencial de la ciudad de Jaguarão, bordes de marcación (magenta) en iteraciones 1, 20 y 40 respectivamente.
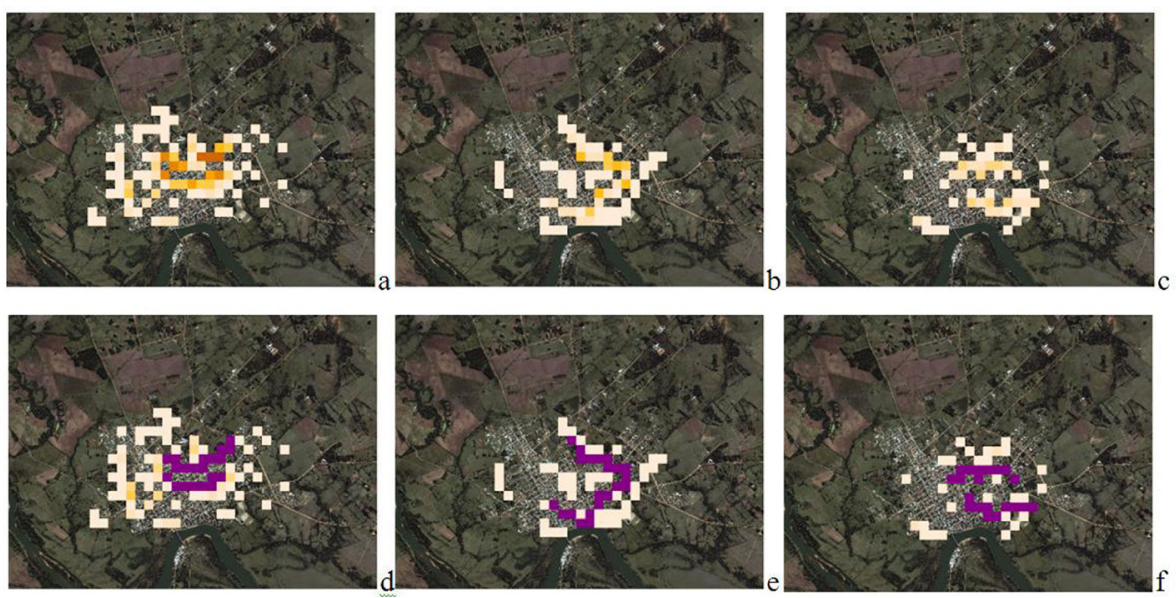

Fuente: Elaboración propia. 


\section{BIBLIOGRAFÍA}

- Alberti, M. (1999). "Modeling the urban ecosystem: a conceptual framework". En Environment and Planning B - Planning and Design v. 26. London: Pion. pp. 605-630.

- Almeida, C. M.; Câmara, G.; Monteiro, A. M. (2007). Geoinformação em urbanismo: cidade real $\mathrm{X}$ cidade virtual. São Paulo: Oficina de Textos.

- Axelrod, R. (1997). "Advancing the art of simulation in the social sciences. International Conference on Computer Simulation and the Social Sciences". Cortona, Italy. 12 p. [Disponible en: www.santafe.edu ]

- Bayón, D.; Gasparini, P. (1977). Panorámica de la arquitectura Latinoamericana. Barcelona: Blume-Unesco.

- Batty, M. (1998). "Urban evolution on the desktop: simulation with the use of extended cellular automata". Environment and Planning A, v. 30. p. 1943-1967.

- Batty, M. (2009). "Urban Modelling". En International Encyclopedia of Human Geography. Editors-in-Chief Rob Kitchin and Nigel Thrift. Disponible en: http:// www.casa.ucl.ac.uk/andrew/repastmodels/presentations/Urban-Modelling-Revised1.pdf

- Benevolo, L. (1975). Diseño de la ciudad. Vol. 1 a 5. Barcelona: GG, 1977.

- Burrough, P. A. (1998). Geocomputation: a primer. London: John Wiley \& Sons Ltd.

- Echenique, M. (Org.) (1975). Modelos matemáticos de la estructura urbana: aplicaciones en América Latina. Buenos Aires: SIAP.

- Greco, A. (2002). "A nova ciência de Stephem Wolfran". Folha de São Paulo Mais! 30 de junio de 2002. p. 20-22.

- Johnson, S. (2003). Emergência: a vida integrada de formigas, cérebros, cidades, softwares. Tradução de Maria C. P. Dias. Rio de Janeiro: Jorge Zahar Ed. 
- Krafta, R. (1994). "Modelling Intraurban configurational development". Environment and Planning B: Planning and Design, v. 21. London: Pion. p. 67-82.

- Krafta, R. (2009). Análise espacial urbana: aplicações na região metropolitana de Porto Alegre. Porto Alegre: Editorial UFRGS.

- Maddox, J. (1983). "Simulating the Replication of Life". Nature n. 305. Disponible en www.StephemWolfram.com

- O'Sullivan, D.; Torrens, P. M. (2000). Cellular models of urban systems. London: Casa, UCL.

- Peres, O. M.; Polidori, M. C. (2009). "Modelos Urbanos baseados em Autômatos Celulares: integrando ambiente natural e o crescimento urbano" En Anais do WCAMA - I Workshop de Computação Aplicada à Gestão do Meio Ambiente e Recursos Naturais, Bento Gonçalves, RS.

- Peres, O. M.; Polidori, M. C. (2010). "O software SACl e o crescimento urbano integrado ao ambiente natural" En Anais do Simpósio I Computers on the beach, Niterói.

- Peres, O. M.; Polidori, M. C. (2011). "Simulação de crescimento, morfologias e o papel da hidrografia na dinâmica de expansão urbana" en XIII Conferência Iberoamericana de Sistemas de Informações Geográficas. Toluca - México. Toluca, México: Editorial UAEM

- Peres, O. M.; Polidori, M. C. (2010) "Modelos urbanos baseados em autômatos celulares: integrando ambiente natural e o crescimento urbano" en Anais do XXIX Congresso da Sociedade Brasileira de Computação. Bento Gonçalves: SBC, 2009.

- Polidori, M. C. (2004). Crescimento urbano e ambiente: um estudo exploratório sobre as transformações e o futuro da cidade. Tese (Doutorado em Ecologia) Universidade Federal do Rio Grande do Sul. Porto Alegre: UFRGS-PPGECO.

- Polidori, M. C. (2003). "Simulação do Ambiente da Cidade". Anais X Encontro Nacional da ANPUR. Belo Horizonte: ANPUR. 
- Polidori, M. C., Granero, J. e Krafta, R. (2001). Software Medidas Urbanas [apoio FAPERGS]. Pelotas: Laboratório de Geoprocessamento da UFPel.

- Polidori, M. C.; Bachilli, G. C. (2007). "Speed, emergence and urban growth". XIII Conferência do ISUF - ISUF 2007 - International Seminar on Urban Form. Ouro Preto: UFMG / ISUF. 14 p.

- Portugali, J. (1997). "Self-organizing cities". Futures, v. 29 nº 4/5. Great Britain: Elsevier Science.

- Saraiva, M.; Polidori, M. C.; Peres, O. M.; Toralles, C. P. (2012). Software CityCell - Urban Growth Simulator. Pelotas: Laboratório de Urbanismo da UFPel.

- Saraiva, M.; Polidori, M. C. (2011). "Simulando crescimento urbano com apoio em SIG, grafos e autômatos celulares". Anais da XIII Conferência Iberoamericana de Sistemas de Informações Geográficas. Toluca, México: Editora da UAEM.

- Torrens, P. (2010a). "How cellular models of urban systems work (1. theory)". Working paper series. Paper 28. Londres: CASA, UCL. Disponible en http:// www.casa.ucl.ac.uk/publications/workingPaperDetail.asp?ID=28

- Torrens, P. (2000b). How land-use-transportation models work. London: Casa, UCL. Disponible en: http://www.casa.ucl.ac.uk/working_papers.htm

- Villaça, Flávio (2001) Espaço intra-urbano no Brasil. São Paulo: Studio Nobel e FAPESP, Lincoln Institute.

- Ward, D.P.; Murray, A.T.; Phinn, S.R. (2000). "A stochastically constrained cellular model of urban groth". Computers, Environment and Urban Systems v. 24. pp. 539-558. Disponible: www.elsevier.com/locate/compenvurbsys ]

- Wilson, A. J. (1985): Mathematical Methods in Human Geography and Planning. Great Britain: John Wiley \& Publishers.404 p.

- Wolfran, S. (1983). "The statistical mechanics of cellular automata". Review of Modern Physics, 55:601-643. Disponible en: http://www.stephenwolfran.com/ publications/articles/ca/ 